\title{
Latest results from the LHCf experiment
}

\author{
T. Suzuki', ${ }^{a}$ O. Adriani, ${ }^{b c}$ E. Berti, ${ }^{b c}$ L. Bonechi, ${ }^{b}$ M. Bongi, ${ }^{b c}$ R. D ' Alessandro, ${ }^{b c}$ M. \\ Haguenauer, ${ }^{e}$ Y. Itow, ${ }^{f g}$ T. Iwata, ${ }^{a}$ K. Kasahara, ${ }^{h}$ Y. Makino, ${ }^{f}$ K. Masuda, ${ }^{f}$ E. \\ Matsubayashi, ${ }^{f}$ H. Menjo, ${ }^{i}$ Y. Muraki, ${ }^{f}$ P. Papini, ${ }^{b}$ S. Ricciarini, ${ }^{b d}$ T. Sako, ${ }^{f g} \mathbf{N}$. \\ Sakurai, ${ }^{j}$ K. Sato,${ }^{f}$ M. Shinoda, ${ }^{f}$ T. Tamura, ${ }^{k}$ A. Tiberio, ${ }^{b c}$ S. Torii, ${ }^{h}$ A. Tricomi ${ }^{l m}$ W. \\ C. Turner, ${ }^{n}$, M. Ueno, ${ }^{f}$ Q. D. Zhou ${ }^{f}$ \\ ${ }^{a}$ Faculty of Science and Engineering, Waseda University - Tokyo, Japan \\ ${ }^{b}$ INFN Section of Florence - Florence, Italy \\ ${ }^{c}$ University of Florence - Florence, Italy \\ ${ }^{d}$ IFAC-CNR - Florence, Italy \\ ${ }^{e}$ Ecole-Polytechnique - Palaiseau, France \\ ${ }^{f}$ Institute for Space-Earth Environmental Research - Nagoya, Japan \\ ${ }^{g}$ Kobayashi-Maskawa Institute for the Origin of Particles and the Universe, Nagoya University - \\ Nagoya, Japan \\ ${ }^{h}$ Research Institute for Science and Engineering, Waseda University - Tokyo, Japan \\ ${ }^{i}$ Graduate School of Science, Nagoya University - Nagoya, Japan \\ j Tokushima University, Tokushima, Japan \\ ${ }^{k}$ Kanagawa University - Kanagawa, Japan \\ ${ }^{l}$ INFN Section of Catania - Catania, Italy l University of Catania - Catania, Italy \\ ${ }^{m}$ University of Catania - Catania, Italy \\ ${ }^{n}$ LBNL - Berkeley, California, USA \\ E-mail: stakuyadaoni.waseda.jp
}

The LHC forward (LHCf) is a small experiment at the LHC designed to measure neutral particles emitted in the very forward region (pseudo-rapidity $\eta>8.4$ ). It has measured proton-proton collisions at $\sqrt{s}=0.9,2.76,7$ and $13 \mathrm{TeV}$, and proton-lead collisions at $\sqrt{s_{N N}}=5.02$ and $8.16 \mathrm{TeV}$. Its main purpose is to provide precise measurements of the production spectra of these forward particles, in order to calibrate hadronic interaction models used in air shower observations. In this paper we will present the current status of the LHCf experiment, regarding in particular collected data and analysis results, as well as future prospects.

38th International Conference on High Energy Physics

3-10 August 2016

Chicago, USA

* Speaker. 


\section{Introduction}

Many measurements were carried to determine the origin and propagation of high energy cosmic rays $\left(E>10^{18} \mathrm{eV}\right)$, but the answer is still unclear. Due to its low flux, measurements are performed at ground level where cosmic rays interact with the atmosphere and developped into extensive air showers. Properties of the primary particle are reconstructed with the help of Monte Carlo (MC) calculations. Since interactions between cosmic rays and the atmosphere are dominated by soft (non-perturbative) QCD, results differ from model to model and become the main source of uncertainties in air shower measurements. The lack of experimental data from accelerator experiments makes the calibration of interaction models difficult. In soft collisions, a large amount of energy tends to be carried by leading particles emitted in the forward direction. Measurement of the forward region is strongly constrained by tight installation space and high radiation. The LHC forward (LHCf) detectors are small and radiation hard detectors designed to measure such soft inelastic collisions at the LHC. The center-of-mass energy of the LHC is $\sqrt{s}=13$ $\mathrm{TeV}$ for $\mathrm{p}-\mathrm{p}$ collisions, which is equivalent to $10^{16.9} \mathrm{eV}$ in the reference system where the target is at rest. This energy is close to that of high energy cosmic rays.

\section{Set up and detectors}

The LHCf detectors[四] are two small sampling calorimeters installed in the LHC tunnel at $\pm 140 \mathrm{~m}$ on both side from the collision point of ATLAS experiment. Charged particles are swept away by the D1 dipole magnet, and only neutral particles with a pseudo-rapidity $\eta>8.4$ can reach the detector. The two detectors, named Arm1 and Arm2, consist of 2 sampling calorimeters made from 22 tungsten plates and 16 GSO scintillators (plastic scintillators before 2014). The total thickness of the calorimeter is $28 \mathrm{~cm}$, which is equivalent to $44 X_{0}$ or $1.6 \lambda_{I}$. The transverse sizes of the calorimeter are $20 \times 20 \mathrm{~mm}^{2}$ and $40 \times 40 \mathrm{~mm}^{2}$ for Arm1, $25 \times 25 \mathrm{~mm}^{2}$ and $32 \times 32 \mathrm{~mm}^{2}$ for Arm2. Energy resolution is better than $5 \%$ for photons above $100 \mathrm{GeV}$ and about $40 \%$ for hadrons above $500 \mathrm{GeV}$. The position of the incident particle is reconstructed using $4 x y$ hodoscopes. Position layers are made from very thin $\left(1 \mathrm{~mm}^{2}\right.$ cross section) GSO scintillators (plastic scintillating fibers before 2014) for Arm1, and from $160 \mu \mathrm{m}$ read-out pitch silicon microstrip detectors for Arm2. Detail performance are described in [, 目]

\section{Analysis and results}

Because LHCf requires low luminosity and high $\beta^{*}$ (applied in some circumstances), measurements were carried under special conditions: in 2009-2010 p-p collisions at $\sqrt{s}=0.9$ and 7 $\mathrm{TeV}$, in $2013 \mathrm{p}-\mathrm{p}$ at $\sqrt{s}=2.76 \mathrm{TeV}$ and $\mathrm{p}-\mathrm{Pb}$ at $\sqrt{s_{N N}}=5.02 \mathrm{TeV}$, in $2015 \mathrm{p}-\mathrm{p}$ at $\sqrt{s}=13 \mathrm{TeV}$, and in $2016 \mathrm{p}-\mathrm{Pb}$ at $\sqrt{s_{N N}}=5.02$ and $8.16 \mathrm{TeV}$. We will present the latest results of $\mathrm{p}-\mathrm{p}$ at $\sqrt{s}=7$ $\mathrm{TeV}$ and $\mathrm{p}-\mathrm{Pb}$ at $\sqrt{s_{N N}}=5.02 \mathrm{TeV}$, and some preliminary results from $\mathrm{p}-\mathrm{p}$ at $\sqrt{s}=13 \mathrm{TeV}$. Spectra of photon, $\pi^{0}$ and neutron for $\mathrm{p}-\mathrm{p}$ collisions at $\sqrt{s}=7 \mathrm{TeV}$ and measurement of the nuclear modification factor for $\mathrm{p}-\mathrm{Pb}$ collisions at $\sqrt{s_{N N}}=5.02 \mathrm{TeV}$ were already presented, and results can be

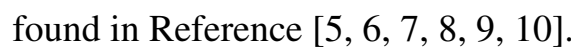




\subsection{Results from Run1 (2009-2013)}

Feynman suggested that the production cross section of secondary particles as a function of Feynman-x $\left(x_{F}=2 p_{z} / \sqrt{s}\right)$ were independent of the incident energy in the forward region. [团]. This is called the Feynman scaling; the $x_{F}$ distribution $\left(\left(x_{F} / \sigma_{\text {inel }}\right)\left(d \sigma / d x_{F}\right)\right)$ is independent of $\sqrt{s}$ for $x_{F}>0.2$. The scaling allows us to extrapolate the spectra measured at LHC to higher cosmic rays energy region. In Fig. I, we compare the $x_{F}$ distributions of $\pi^{0}$ production in two $p_{T}$ ranges: $0.0<p_{T}<0.2$ and $0.2<p_{T}<0.4 \mathrm{GeV} / \mathrm{c}$ for $\mathrm{p}-\mathrm{p}$ collisions at $\sqrt{s}=2.76$ and $7 \mathrm{TeV}$. LHCf results confirm that the scaling holds at $20 \%$ level[ए]].

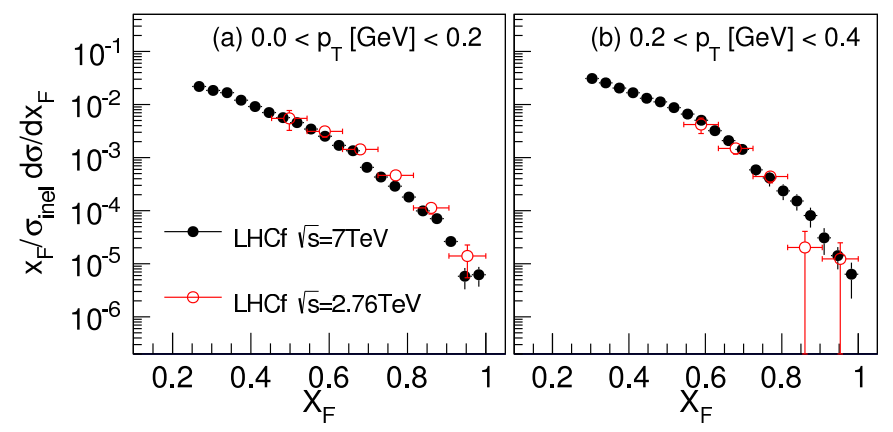

Figure 1: $\pi^{0}$ yield in each $p_{T}$ range as a function of $x_{F}$. Red open circles and black filled circles indicate measurements in $\mathrm{p}-\mathrm{p}$ collisions at $\sqrt{s}=2.76$ and $7 \mathrm{TeV}$, respectively [U]].

Fig.】 shows the nuclear modification factor as a function of $p_{T}$ and $y_{l a b}$. Nuclear modification factor is defined with the following formula: $R_{p P b}=\frac{\sigma_{\text {inel }}^{P p}}{<N_{\text {coll }}>\sigma_{\text {inel }}^{P P D}} \frac{E d^{3} \sigma^{P P b} / d p^{3}}{E d^{3} \sigma^{p p} / d p^{3}}$.

The cross section for $\mathrm{p}-\mathrm{p}$ at $\sqrt{s}=5.02 \mathrm{TeV}$ was interpolated from $\sqrt{s}=2.76$ and $7 \mathrm{TeV}$ data[W]]. Given the large uncertainties, all models are in good agreement with the measurements, predicting a decrease of the production rate in the case the target nucleus is $\mathrm{Pb}$.

\subsection{Prelimininary analysis of Run2 (p-p collision at $\sqrt{s}=13 \mathrm{TeV}$ )}

LHC provided $\mathrm{p}-\mathrm{p}$ collisions at $\sqrt{s}=13 \mathrm{TeV}$ with a luminosity of $10^{29} \mathrm{~cm}^{-2} \mathrm{~s}^{-1}$ and $\beta^{*}$ from 10 to $13 \mathrm{~m}$. LHCf took data during 32 hours $\left(8^{\text {th }}\right.$ to $12^{\text {th }}$ of June 2015) under these special conditions. This operation was achieved with the ATLAS collaboration. The trigger signals from LHCf were sent to ATLAS that allowed us to record combined data. This common operation is expected to classify events based on the nature of the processes: diffractive and non-diffractive. Selecting diffractive events will help to improve the study of soft collisions[12]. Fig.[1] shows the invariant mass distribution of two photons event which is necessary for energy calibration. The mass of $\pi^{0}$ and $\eta$ are clearly reconstructed at around 135 and $548 \mathrm{MeV}$, respectively. In Fig. 国, the energy spectra of the reconstructed photons from data are plotted with those of MC predicted by various interaction models in two rapidity regions: $8.99<\eta<8.81$ and $\eta>10.94$. Post-LHC interaction models were used and EPOS-LHC agrees with measured data well.

\section{Conclusions and future plans}

The LHCf experiment was designed to measure neutral particles emitted in $\eta>8.4$. We 


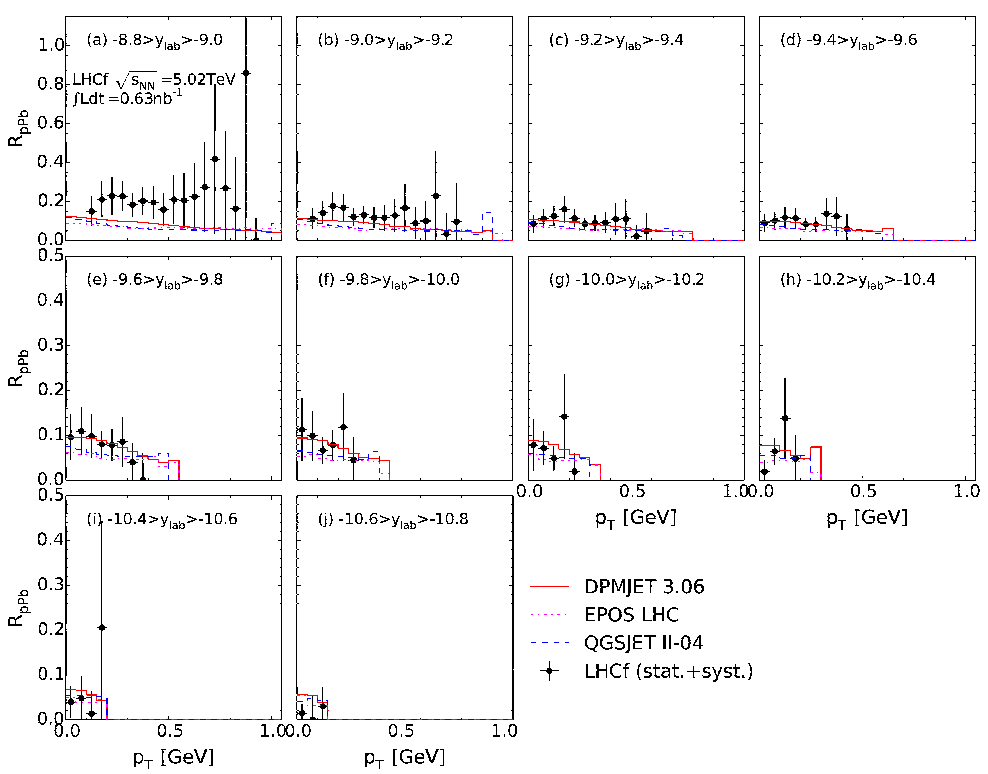

Figure 2: Nuclear modification factor of $\pi^{0}$ in various $p_{T}$ ranges as a function of $y_{l} a b$. Filled black circles are the LHCf measurements with error bars including statistical and systematic uncertainties. Other colored lines are the MC predictions by hadronic interaction models[ए]].

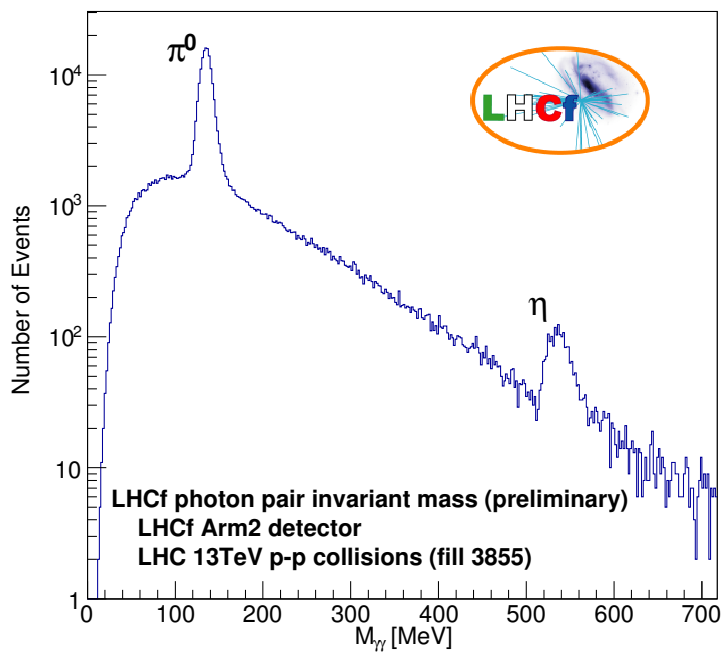

Figure 3: Photon pair invariant mass obtained from $\mathrm{p}-\mathrm{p}$ collision at $\sqrt{s}=13 \mathrm{TeV}$ using Arm2. The mass of $\pi^{0}$ and $\eta$ are clearly reconstructed at $\sim 135 \mathrm{MeV}$ and $\sim 548 \mathrm{MeV}$, respectively. 

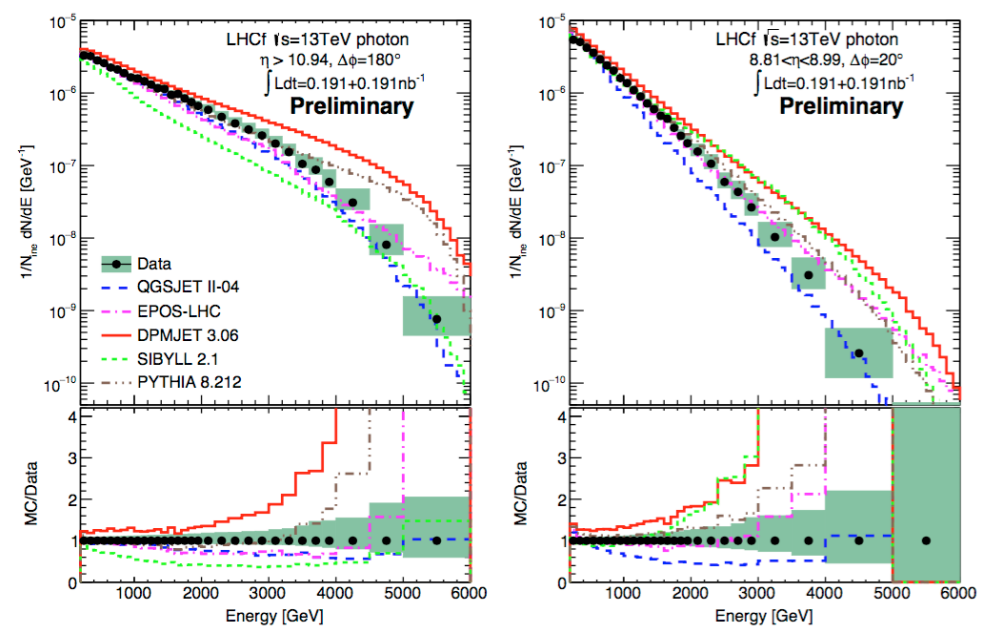

Figure 4: Comparison of the single photon energy spectra between experimental data and MC calculations. Top panels show the spectra and bottom panels show the ratios of MC results(colored) to experimental data(black).

measured $\mathrm{p}-\mathrm{p}$ and $\mathrm{p}-\mathrm{Pb}$ collisions at LHC with various collision energies and showed that no model can perfectly explain the experimental data. Measurements of energy and $p_{T}$ spectra of the neutral particles produced in the very forward region can therefore be used to calibrate models. Further study with ATLAS collaboration can help to identify different types of events, e.g. diffractive and non diffractive. In October 2016, LHC took data with $\mathrm{p}-\mathrm{Pb}$ collisions at $\sqrt{s}=8.16 \mathrm{TeV}$. In 2017, the LHCf Arm1 detector will be used as the RHICf detector to measure polarized proton-proton collisions at $\sqrt{s}=510 \mathrm{GeV}[\mathbb{[ 1 3}]$.

\section{References}

[1] O. Adriani et al., JINST 3 (2008) S08006.

[2] T. Mase et al., Nucl. Instrum. Meth. A671 (2012) 129.

[3] K. Kawade et al., JINST 9 (2014) P03016.

[4] R. Feynman, Phys. Rev. Lett. 23 (1969) 1415.

[5] O. Adriani et al., Phys. Lett. 703 (2011) 128.

[6] O. Adriani et al., Phys. Lett. 715 (2012) 298.

[7] O. Adriani et al., Phys. Rev. D86 (2012) 092001.

[8] O. Adriani et al., Phys. Rev. C89 (2014) 065209.

[9] O. Adriani et al., Phys. Lett. B750 (2015) 360.

[10] O. Adriani et al., Phys. Rev. C89 (2014) 065209.

[11] O. Adriani et al., Phys. Rev. D94 (2016) 032007.

[12] Q. D. Zhou et al., arXiv:1611.07483 [hep-ex] (2016).

[13] Y. Itow et al., arXiv:1409.4860 [physics.ins-det] (2014). 